\title{
On Partially Ordered Real Involutory Algebras
}

\author{
S. Albeverio - Sh. A. Ayupov • R. A. Dadakhodjayev
}

Received: 26 November 2004 / Accepted: 21 December 2005 /

Published online: 15 November 2006

(C) Springer Science + Business Media B.V. 2006

\begin{abstract}
In this paper we study real $O^{*}$-algebra, on the hermitian elements of which a partial order exists which is compatible with the algebraic structure. Such algebras occur in the axiomatic approach to the description of the space of random variables (observables) in quantum probability theory. We study the relations between these so called real $O^{*}$-algebras and their complexification, and also their Jordan structure. Our main result is the theorem on the representation of abstract real $O^{*}$-algebras as algebras of locally measurable (unbounded) operators affiliated with a real von Neumann algebra on a Hilbert space.
\end{abstract}

\footnotetext{
S. Albeverio $(\varangle)$

Institut für Angewandte Mathematik, Universität Bonn, Wegelerstr. 6, 53115 Bonn, Germany e-mail: albeverio@uni-bonn.de
}

S. Albeverio

SFB 611, Universität Bonn, Poppelsdorfer Allee 82, 53115 Bonn, Germany

S. Albeverio

BiBoS, Universität Bielefeld, Postfach 1001 31, 33501 Bielefeld, Germany

S. Albeverio

CERFIM, Casella Postale 1132, Via F. Rusca 1, 6601 Locarno, Switzerland

S. Albeverio

Acc. Arch., Università della Svizzera Italiana, Via Largo Bernasconi 2, 6850 Mendrisio, Italy

S. A. Ayupov · R. A. Dadakhodjayev

Institute of Mathematics, Uzbekistan Academy of Science, F. Hodjaev str. 29, 700143,

Tashkent, Uzbekistan

S. A. Ayupov

e-mail: e_ayupov@hotmail.com

R. A. Dadakhodjayev

e-mail: Rashidkhon@mail.ru 
Key words ordered algebras • real $O^{*}$-algebras - complexification • involutive *-anti-automorphism $\cdot$ discrete $O^{*}$-factors $\cdot$ locally measurable operators.

Mathematics Subject Classifications (2000) 46L45 - 46L50.

\section{Introduction}

This work is devoted to an axiomatic approach to the description of the space of random variables in quantum probability theory in the framework of real involutory algebras. Unlike the $C^{*}$-algebras (or $W^{*}$-algebras) models dealing with bounded observables, our approach includes rather wide classes of unbounded observables and in the particular case of abelian *-algebras it describes the algebra of all random variables in the classical probability theory.

Such ideas were firstly suggested in the paper of T. A. Sarymsakov and M. Sh. Goldshtein [12], where the notion of a partially ordered complex involutory algebra was introduced, such that on its hermitian elements a partial order is defined which is compatible with the algebraic operations. These algebras were called $O^{*}$ algebras. Later the structure of these algebras was investigated and relations with operator algebras ( $C^{*}$-algebras, $W^{*}$-algebras, $A W^{*}$-algebras, etc.) were established. In particular a theorem on the representation of abstract $O^{*}$-algebras as algebras of measurable operators affiliated with von Neumann algebras on Hilbert spaces was proved (see the monograph [13] for details). There are also many application of $O^{*}$-algebras in the non commutative probability theory and the theory of stochastic processes. In this approach the hermitian elements of an $O^{*}$-algebra $E$ are interpreted as random variables (or observables) which are unbounded in general, the projections of the $O^{*}$-algebra $E$ (i.e., self-adjoint idempotents) - as events, and states on the algebra of bounded elements of $E$ as probability measures or quantum states.

The theory of $O^{*}$-algebras as well as the theory of operator algebras $\left(C^{*}\right.$-, $W^{*}$ algebras, etc.) is usually developed over the field of complex numbers. But from the probabilistic point of view it is more natural to consider real algebras. Nevertheless as it was mentioned in the recent monograph of Li Bing-Ren [11] "up to now the theory of operator algebras over the field of real numbers seems not to be introduced systematically and sufficiently". So in the mentioned book the author gives a systematic discussion of real operator algebras and sets up the fundamentals of real Banach spaces and Hilbert spaces, real Banach algebras, real $C^{*}$-algebras and $W^{*}$-algebras, and other structures over the reals. Later an analogue of the theory of $A W^{*}$-algebras (Kaplansky algebras) over the reals was developed in the papers $[1,2,6,7]$.

This is the starting point of the present paper which in devoted to the real counterpart of the theory of $O^{*}$-algebras. Similar to the case of $A W^{*}$-algebras $[1,2]$ some properties of real $O^{*}$-algebras are the same as of complex $O^{*}$-algebras, but there are also properties which completely differ from the complex case. In particular we prove in Section 2 that the set of all symmetric (hermitian) elements of a real $O^{*}$-algebra forms an ordered Jordan algebra [5, 13] as in the case of complex $O^{*}$-algebras. We show that if the complexification of a real *-algebra $E$ is a (complex) $O^{*}$-algebra then $E$ itself is a real $O^{*}$-algebra. On the other hand we 
give examples of real $O^{*}$-algebras complexification of which are not $O^{*}$-algebras. We also study the subalgebra of bounded elements of a real $O^{*}$-algebra and prove that it is a normed ${ }^{*}$-algebra but may not be a real $C^{*}$-algebra (while the bounded part of complex $O^{*}$-algebras always forms a $C^{*}$-algebra) (Section 3). In Section 4 we give necessary and sufficient conditions for two elements to commute in terms of symmetrized (i.e., Jordan) products and prove that in real $O^{*}$-algebras there is no Heisenberg representation of canonical commutation relations, i.e., scalar multiples of the identity can not be equal to the commutator of two hermitian elements. We completely describe discrete real $O^{*}$-factors and prove that they are isomorphic to the algebra $B(H)$ of all bounded linear operators on either a real or a quaternionic Hilbert space $H$. In Section 5 we construct the most general examples of real $O^{*}$ algebras $*^{*}$-algebras of all measurable and locally measurable operators affiliated with a real von Neumann algebra, and prove the main result of the paper: under rather natural conditions any abstract real $O^{*}$-algebra can be embedded into the real $O^{*}$-algebra of all locally measurable operators affiliated with a real von Neumann algebra.

Remark A different notion of an $O^{*}$-algebra as a *-algebra of closable operators defined on a dense subspace in a Hilbert space was considered by K. Schmüdgen, A. Inoue and collaborators (see e.g., $[4,10,14]$ ). The $O^{*}$-algebras considered by T.A. Sarymsakov and M.Sh. Goldshtein [12] (the complex case) and in this paper (the real case) are essentially different even for concrete (not abstract) $O^{*}$-algebras of unbounded operators on a Hilbert space, because unlike $O^{*}$-algebras in the sense of $[4,10,14]$, our $O^{*}$-algebras of measurable or locally measurable operators affiliated with a von Neumann algebra (see Theorems 5.4 and 5.5. below) are not defined on a common dense subspace of the underlying Hilbert space.

\section{Real O*-algebras}

Let $E$ be an associative ${ }^{*}$-algebra over the field of reals $\mathbb{R}, E_{h}=\left\{x \in E: x^{*}=x\right\}-$ the space of all hermitian elements in $E$.

Definition 1 A partial order $\geq$ on $E_{h}$ is said to be compatible with the algebraic operations, if it satisfies the following conditions:

1) $x \geq y \Rightarrow x+z \geq y+z$, for all $z \in E_{h}$;

2) $x \geq y \Rightarrow \lambda x \geq \lambda y$, for all $\lambda \in \mathbb{R}, \lambda \geq 0$;

3) If $x \geq 0, y \geq 0, \quad x y=y x$ then $x y \geq 0$;

4) $x^{*} x \geq 0$ for all $x \in E$ and $x^{*} x=0$ implies $x=0$.

Definition 2 A real involutory algebra $E$ with the identity $\mathbf{I}$ is called a real $O^{*}$ algebra, if $E_{h}$ admits a partial order compatible with the algebraic operations and such that

(a) If $\left\{x_{\alpha}\right\}$ is any monotone increasing net of hermitian elements bounded above, then there exists $x=\sup _{\alpha} x_{\alpha}$, and if for $y \in E x_{\alpha} y=y x_{\alpha}$ for all $\alpha$, then $x y=y x$;

(b) If $\tilde{\mathrm{E}}$ is any maximal commutative subalgebra in $E$ then $\tilde{\mathrm{E}}_{h}=\left\{x \in \tilde{\mathrm{E}}: x^{*}=x\right\}$ is a lattice with respect to the induced partial order. 
Example 1 Let $E_{0}$ be the algebra of all measurable real functions on a measure space $(\Omega, \Sigma, \mu)$, i.e., $E_{0}=L^{0}(\Omega, \Sigma, \mu)$ (or more generally let $E_{0}$ be a semi-field in the sense of [11]). Then $E_{0 h}=E_{0}$ and $E_{0}$ is a commutative real $O^{*}$-algebra.

Example 2 Let $E_{0}$ be as above. Consider the space

$$
E=E_{0}+i E_{0}=\left\{a+i b, a, b \in E_{0}\right\}
$$

and equip $E$ with coordinate-wise linear operations, the product

$$
\left(a_{1}+i b_{1}\right)\left(a_{2}+i b_{2}\right)=\left(a_{1} a_{2}-b_{1} b_{2}\right)+i\left(a_{1} b_{2}+b_{1} a_{2}\right)
$$

and the involution

$$
(a+i b)^{*}=a^{*}-i b^{*} .
$$

Then $E$ is a both a complex [13] and a real commutative $O^{*}$-algebra.

Example 3 Any complex $O^{*}$-algebra $[12,13]$ is a real $O^{*}$-algebra at the same time. Moreover in this case the second part of the axiom (4), i.e., $x^{*} x=0 \Rightarrow x=0$ is redundant - it follows from other axioms (see [13, Ch. IV, §2, Proposition 1]). In particular any von Neumann algebra is an example of a real $O^{*}$-algebra.

Example 4 Any real von Neumann algebra $\Re$ is a real $O^{*}$-algebra.

Remark Unlike the complex case the axiom $x^{*} x=0 \Rightarrow x=0$ is not redundant in the real case as the following example shows. Consider the real $O^{*}$-algebra $E_{0}$ from the Example 1. Put $E=E_{0}+i E_{0}$, equip $E$ with coordinate-wise linear operations, involution $(a+i b)^{*}=a-i b$ and the multiplication

$$
\left(a_{1}+i b_{1}\right)\left(a_{2}+i b_{2}\right)=a_{1} a_{2}+i\left(a_{1} b_{2}+b_{1} a_{2}\right)
$$

(i.e., $\left.i^{2}=0\right)$. Then it is clear that $E_{h}=E_{0}$ and all the axioms (1)-(3), (a),(b) are fulfilled (since $E$ is commutative). Moreover for all $x=a+i b$ we have

$$
x^{*} x=a^{2}+i(a b-b a)=a^{2} \geq 0 .
$$

But $x^{*} x=0$ does not imply that $x=0$, since for $x=i b, b \neq 0, b \in E_{0}$, we have $x^{*} x=0$.

Now let as consider the relation between real $O^{*}$-algebras and partially ordered Jordan algebras in the sense of [5, 13]. In the monograph [13] it was proved that the hermitian part of any (complex) $O^{*}$-algebra is a partially ordered Jordan algebra $O J$-algebra. A similar result is true also for real $O^{*}$-algebras.

Let $E$ be a real $O^{*}$-algebra, $E_{h}$ - its hermitian part. Consider on $E_{h}$ the symmetrized (Jordan) product $x \circ y=\frac{1}{2}(x y+y x)$, so $\left(E_{h}, \circ\right)$ becomes a Jordan algebra.

Recall that two elements $a, b$ in a Jordan algebra $J$ are said to be compatible (or simultaneously observable, denoted as $a \leftrightarrow b$ ) if the Jordan subalgebra $J(a, b)$ generated by these elements is strongly associative, that is

$$
\{x, z, y\}=(x \circ z) \circ y-x \circ(z \circ y)=0
$$


for all $x, y \in J(a, b)$ and any $z \in J$, i.e., any two elements of $J(a, b)$ operator commute. It is known [13] that $a \leftrightarrow b$ if and only if all elements $a, b, a^{2}, b^{2}, a b$ pairwise operator commute.

In the particular case where the Jordan products is induced by an associative product as $x \circ y=\frac{1}{2}(x y+y x)$ it is easy to see that

$$
\{a, c, b\}=a \circ(c \circ b)-(a \circ c) \circ b=\frac{1}{4}[c,[a, b]]
$$

where $[x, y]=x y-y x$.

Proposition 2.1 Let $E$ be a real $O^{*}$-algebra, $E_{h}$ - its hermitian part. For $a, b \in E_{h}$ the following conditions are equivalent

(1) $[a, b]=0$;

(2) $a \leftrightarrow b$ in $\left(E_{h}, \circ\right)$.

Proof

$(1) \Rightarrow(2): \quad$ Let $a b=b a$, then it is clear that all elements $a, b, a^{2}, b^{2}, a b$ pairwise operator commute. For example $\left\{a, c, b^{2}\right\}=\frac{1}{4}\left[c,\left[a, b^{2}\right]\right]=0$ because $\left[a, b^{2}\right]=0$. Thus as it was mentioned above $a \leftrightarrow b$.

(2) $\Rightarrow(1)$ : Let $a \leftrightarrow b$, i.e., all elements $a, b, a^{2}, b^{2}, a b$ mutually operator commute.

In particular

$$
\begin{gathered}
{[c,[a, b]]=4\{a, c, b\}=0 \text { for all } c \in E_{h}} \\
{\left[c,\left[a^{2}, b\right]\right]=4\left\{a^{2}, c, b\right\}=0 \text { for all } c \in E_{h} .}
\end{gathered}
$$

From Eq. 2 we have that $[a, b]$ commutes with each hermitian element in $E$, and since $a \in E_{h}$ we have

$$
\begin{aligned}
{\left[a^{2}, b\right]=} & a^{2} b-b a^{2}=a^{2} b-a b a+a b a-b a^{2}=a(a b-b a) \\
& +(a b-b a) a=a[a, b]+[a, b] a=2 a[a, b] .
\end{aligned}
$$

Therefore Eq. 3 implies that for any $c \in E_{h}$ :

$$
\begin{aligned}
0 & =\left[c,\left[a^{2}, b\right]\right]=[c, 2 a[a, b]]=2 c a[a, b]-2 a[a, b] c \\
& =2 c a[a, b]-2 a c[a, b]=2[c, a][a, b] .
\end{aligned}
$$

In particular putting $a=c$ we obtain

$$
0=[b, a][a, b]=[a, b]^{*}[a, b] .
$$

Now the axiom (4) of real $O^{*}$-algebras implies that $[a, b]=0$. The proof is complete.

Theorem 2.2 The hermitian part $E_{h}$ of an arbitrary real $O^{*}$-algebra $E$, equipped with the symmetrized product forms an OJ-algebra. Conversely, let the hermitian part $E_{h}$ of a real ${ }^{*}$-algebra E equipped with the symmetrized product form an OJ-algebra and the let the following condition be hold:

(*) Given any $x \in E, x \neq 0$, there exists $a \in E_{h}, a \neq 0$, such that $x^{*} x=a^{2}$.

Then $E$ is a real $O^{*}$-algebra. 
Proof Let $E$ be a real $O^{*}$-algebra. Consider the Jordan algebra $\left(E_{h}, \circ\right)$. By Proposition 2.1 the compatibility of elements from $\left(E_{h}, \circ\right)$ means their commutativity in $E$. Therefore the validity of the axioms of $O J$-algebras (see [13, Ch. III]) follows from the corresponding axioms (1)-(4) and (a),(b) of real $O^{*}$-algebras and from the fact that any maximal strongly associative subalgebra in $\left(E_{h}, \circ\right)$ is the hermitian part of a maximal abelian *-subalgebra in $E$.

Conversely, suppose that $E$ is a real *algebra such that $\left(E_{h}, \circ\right)$ is an $O J$-algebra and the condition (*) is satisfied. Let us prove that $E$ is a real $O^{*}$-algebra.

First of all note that in this case we also have that the conditions $a \leftrightarrow b$ and $[a, b]=0$ are equivalent: the proof repeats step by step the proof of Proposition 2.1 with the only difference that the implication $[a, b]^{*}[a, b]=0 \Rightarrow[a, b]=0$ is implied by the condition $(*)$. Indeed if $[a, b] \neq 0$, then there exists an element $a \neq 0 a \in E_{h}$, such that $[a, b]^{*}[a, b]=a^{2}$. But since any $O J$-algebra is formally real [13, Ch.III, $\S 3$, Corollary of Theorem 1], one has that $a^{2} \neq 0$.

Now, all the axioms of the real $O^{*}$-algebra follow from the corresponding axioms of the $O J$-algebra. The proof is complete.

Theorem 2.2 implies that all of results concerning the hermitian elements of real $O^{*}$ algebras, the lattice of projections, etc. easily follow from the corresponding results on $O J$-algebras similar to the case of complex $O^{*}$-algebras [13, Ch. IV]. In particular:

- Projections $\left\{e \in E: e^{2}=e=e^{*}\right\}$ of real $O^{*}$-algebras form a complete orthomodular lattice in the induced order;

- The spectral theorem for hermitian elements of real $O^{*}$-algebras is valid i.e., given any hermitian element $x \in E_{h}$ there exists a spectral family of projections $\left\{e_{\lambda}^{x}, \lambda \in \mathbb{R}\right\}$ such that $x=\int_{-\infty}^{+\infty} \lambda d e_{\lambda}^{x}$ where the convergence of the integral means the order convergence (see for details [13, Ch. III]);

- Given any $a \in E$ the map $x \rightarrow a^{*} x a$ is positive and normal, i.e., $x \geq 0 \Rightarrow a^{*} x a \geq$ 0 , and $x_{\gamma} \downarrow 0 \Rightarrow a^{*} x_{\gamma} a \downarrow 0$, etc.

Given a real $O^{*}$-algebra $E$ consider its complexification $F=E+i E$ which is clearly a complex $*$-algebra. A natural question arises: is there any partial order on $F_{h}$ such that $F$ becomes a (complex) $O^{*}$-algebra?

It is known [13] that if such an order exists then it is unique and the cone of positive elements $F^{+}$coincides with the set $\left\{x^{*} x, x \in F\right\}$. The following example shows that the answer to the above question is negative in general.

Example 5 Let $m$ be the commutative algebra of all bounded sequences of real numbers, $c_{0}$ - the subalgebra (ideal) in $m$ consisting of all sequences converging to zero. Put $B=m+i c_{0}$, i.e., $B$ is a real *-algebra of all bounded complex sequences $\left\{\lambda_{n}\right\}$ which are "real at infinity," i.e., $\operatorname{Im} \lambda_{n} \rightarrow 0$ as $n \rightarrow \infty$. Then since $B_{h}=m$ it is clear that $B$ is a commutative real $O^{*}$-algebra. But in [1] it was proved that the $C^{*}$ algebra $B+i B$ is not an $A W^{*}$-algebra. Therefore $B+i B$ is also not an $O^{*}$ algebra, because any $C^{*}$-algebra which is an $O^{*}$-algebra (i.e., an $O C^{*}$-algebra [13]) is necessarily an $A W^{*}$-algebra (see [13, Ch. IV]). 
Now let us consider the converse problem: given a real *-algebra suppose that its complexification admits the $O^{*}$-algebra structure; has the initial real *-algebra the structure of a real $O^{*}$-algebra? The following theorem gives a positive answer to this question.

Theorem 2.3 Let $E$ be an associative real *-algebra with the identity $\mathbf{I}$. Suppose that its complexification $F=E+i E$ admits a partial order such that $F$ is a (complex) $O^{*}$ algebra. Then $E$ is a real $O^{*}$-algebra with respect to the partial order induced on $E_{h}$ from $F_{h}$.

Proof We recall that a (complex) linear map $\alpha: F \rightarrow F$ is called an involutive ${ }^{*}$-antiautomorphism if :

1) $\alpha\left(x^{*}\right)=\alpha(x)^{*}$,

2) $\alpha(x y)=\alpha(y) \alpha(x)$,

3) $\alpha^{2}(x)=x$,

for all $x, y \in M$.

An arbitrary element $x \in F=E+i E$ can be represented as $x=x_{1}+i x_{2}$, where $x_{1}, x_{2} \in E$. Then the canonical involutive ${ }^{*}$-anti-automorphism on $F$ generated by $E$ is defined as

$$
\alpha(x)=x_{1}^{*}+i x_{2}^{*}, \quad x=x_{1}+i x_{2} \in F .
$$

Then it straightforward to check that $\alpha$ is an involutive *-anti-automorphism on $M$ and

$$
E=\left\{x \in F: \alpha(x)=x^{*}\right\} .
$$

Indeed, one has

$$
\begin{aligned}
\alpha\left(x^{*}\right) & =\alpha\left(x_{1}^{*}-i x_{2}^{*}\right)=x_{1}-i x_{2}=\left(x_{1}^{*}+i x_{2}^{*}\right)^{*}=\alpha(x)^{*} . \\
\alpha(x y) & =\alpha\left(\left(x_{1} y_{1}-x_{2} y_{2}\right)+i\left(x_{2} y_{1}+x_{1} y_{2}\right)\right)=\left(x_{1} y_{1}-x_{2} y_{2}\right)^{*}+i\left(x_{2} y_{1}+x_{1} y_{2}\right)^{*} \\
& =\left(y_{1}^{*} x_{1}^{*}-y_{2}^{*} x_{2}^{*}\right)+i\left(y_{1}^{*} x_{2}^{*}+y_{2}^{*} x_{1}^{*}\right)=\left(y_{1}^{*}+i y_{2}^{*}\right)\left(x_{1}^{*}+i x_{2}^{*}\right)=\alpha(y) \alpha(x) . \\
\alpha(\alpha(x)) & =\alpha\left(x_{1}^{*}+i x_{2}^{*}\right)=x_{1}+i x_{2}=x .
\end{aligned}
$$

Further, the real linearity of $\alpha$ is clear. Therefore it is sufficient to show that for $x \in F$

$$
\alpha(i x)=i \alpha(x)
$$

But

$\alpha(i x)=\alpha\left(i\left(x_{1}+i x_{2}\right)\right)=\alpha\left(-x_{2}+i x_{1}\right)=-x_{2}^{*}+i x_{1}^{*}=i\left(x_{1}^{*}+i x_{2}^{*}\right)=i \alpha\left(x_{1}+i x_{2}\right)=i \alpha(x)$.

Finally given any $x=x_{1}+i x_{2} \in F$ we have

$$
x \in E \Leftrightarrow x_{2}=0 \Leftrightarrow x^{*}=x_{1}^{*}=\alpha(x) \text {, i.e. } E=\left\{x \in F: \alpha(x)=x^{*}\right\} .
$$

Consider the hermitian parts $F_{h}$ and $E_{h}$ of $F$ and $E$ respectively. Then

$$
E_{h}=\left\{x \in F: \alpha(x)=x^{*}=x\right\}=\left\{x \in F_{h}: \alpha(x)=x\right\} .
$$

It is clear that the partial order an $E_{h}$ induced from $F_{h}$ is compatible with the algebraic operation in the sense of Definition 1. Note that the condition $x^{*} x=0 \Rightarrow$ 
$x=0$ follows from the corresponding property of complex $O^{*}$-algebras $[13, \mathrm{Ch}$. IV, $\S 2$, Proposition 1]. Now let us check the axioms (a) and (b) of real $O^{*}$-algebras.

(a): Let $\left\{x_{\gamma}\right\}$ be an increasing set of hermitian elements of $E$ bounded from above. Then each $x_{\gamma}$ also belongs to $F$ and $\alpha\left(x_{\gamma}\right)=x_{\gamma}$. Since $F$ is a complex $O^{*}$ algebra, there exists $x=\sup x_{\gamma}$. But any ${ }^{*}$-anti-automorphism is an order isomorphism on $F_{h}$, therefore

$$
\alpha(x)=\alpha\left(\sup x_{\gamma}\right)=\sup \alpha\left(x_{\gamma}\right)=\sup x_{\gamma}=x,
$$

i.e., $x \in E_{h}$ is the least upper bound for $x_{\gamma}$ in $E_{h}$. If given $y \in E_{h}$ one has $x_{\gamma} y=$ $y x_{\gamma}$ for all $\gamma$, then $x y=y x$ also by the corresponding axiom of complex $O^{*}$ algebras.

(b): Let $\tilde{\mathrm{E}}$ be a maximal abelian *-subalgebra in $E$, then it is clear that $\tilde{\mathrm{F}}=\tilde{\mathrm{E}}+\mathrm{i} \tilde{\mathrm{E}}$ is a maximal abelian *-subalgebra in $F$ and $\tilde{\mathrm{E}}=\tilde{\mathrm{F}} \cap E$. By the axiom (II) of complex $O^{*}$-algebras $\tilde{\mathrm{F}}_{h}$ is a lattice, i.e., given any $x, y \in \tilde{\mathrm{F}}_{h}$ there exist $x \vee y$ in $\tilde{\mathrm{F}}_{h}$. Suppose that $x, y \in \tilde{\mathrm{E}}_{h}$, then $x \vee y$ in $\tilde{\mathrm{F}}_{h}$ exists. Since $\alpha$ is an order isomorphism of $F_{h}$ one has

$$
\alpha(x \vee y)=\alpha(x) \vee(y)=x \vee y \text {, i.e., } x \vee y \in \tilde{\mathrm{E}}_{h} .
$$

Similarly $x \wedge y \in \tilde{\mathrm{E}}_{h}$, i.e., $\tilde{\mathrm{E}}_{h}$ is a lattice. Therefore $E$ is a real $O^{*}$-algebra. The proof is complete.

\section{The Subalgebra of Bounded Elements in Real O*-algebras}

It is known $[12,13]$ that in a complex $O^{*}$-algebra the set of bounded elements forms an $O^{*}$-subalgebra and with respect to a certain norm it becomes a $C^{*}$-algebra. In this section we consider a similar notion in real $O^{*}$-algebras.

Definition 3 An element $a$ in a real $O^{*}$-algebra $E$ is said to be order bounded if $a^{*} a \leq \lambda \mathbf{I}$ for an appropriate $\lambda \geq 0, \lambda \in \mathbb{R}$.

Proposition 3.1 Let $E$ be a real $O^{*}$-algebra. Then

(1) An element $a \in E$ is bounded if and only if $a^{*}$ is bounded;

(2) If the elements $a, b \in E$ are bounded, then the elements $\lambda a, a+b, a b$ are also bounded, $\lambda \in \mathbb{R}$.

Proof

(1) Let $a^{*} a \leq \lambda \mathbf{I}$. Since the element $a a^{*}-\lambda \mathbf{I}$ is hermitian, one has $\left(a a^{*}-\lambda \mathbf{I}\right)^{2} \geq 0$, i.e.,

$$
a\left(a^{*} a\right) a^{*}-2 \lambda a a^{*}+\lambda^{2} \mathbf{I}=\left(a a^{*}\right)^{2}-2 \lambda a a^{*}+\lambda^{2} \mathbf{I} \geq 0 .
$$

From $a^{*} a \leq \lambda \mathbf{I}$ follows that $a\left(a^{*} a\right) a^{*} \leq a(\lambda \mathbf{I}) a^{*}=\lambda a a^{*}$ in view of positivity of the map $x \rightarrow a x a^{*}$ (see the note after Theorem 2.2). Therefore $\lambda\left(a a^{*}\right)-$ $2 \lambda a a^{*}+\lambda^{2} \mathbf{I} \geq 0$, i.e., $-a a^{*}+\lambda \mathbf{I} \geq 0$ so $a a^{*} \leq \lambda \mathbf{I}$, i.e., the element $a^{*}$ is also bounded. 
(2) If $a \in E$ is bounded then $\lambda a$ is also bounded for any $\lambda \in \mathbb{R}$. Suppose now that $a, b$ are bounded, i.e., $a^{*} a \leq \lambda \mathbf{I}, b^{*} b \leq \mu \mathbf{I}, \lambda, \mu \in \mathbb{R}, \lambda, \mu \geq 0$. The axiom (4) of real $O^{*}$-algebras implies that $(a-b)^{*}(a-b) \geq 0$, i.e., $a^{*} a+b^{*} b-a^{*} b-$ $b^{*} a \geq 0$, i.e., $a^{*} b+b^{*} a \leq a^{*} a+b^{*} b$. Therefore

$$
\begin{aligned}
(a+b)^{*}(a+b) & =a^{*} a+a^{*} b+b^{*} a+b^{*} b \leq a^{*} a+a^{*} a+b^{*} b+b^{*} b \\
& \leq 2 \lambda \mathbf{I}+2 \mu \mathbf{I}=2(\lambda+\mu) \mathbf{I},
\end{aligned}
$$

i.e., $a+b$ is bounded. Further $(a b)^{*}(a b)=b^{*} a^{*} a b \leq b^{*}(\lambda \mathbf{I}) b=\lambda b^{*} b \leq \lambda \mu \mathbf{I}$, i.e., $a b$ is also bounded. The proof is complete.

Corollary 3.2 The set $A$ of all bounded elements in a real $O^{*}$-algebra $E$ is a real *-subalgebra in $E$, and a solid $O^{*}$-subalgebra.

Indeed $A$ is a real $*$-subalgebra and is solid in $E$, i.e., $0 \leq a \leq b, b \in A, a \in E$ implies that $a \in A$. Therefore the axioms (a), (b) of real $O^{*}$-algebras as well as (1)(4) are inherited from $E$.

Remark From [13, Ch. IV, §3, Proposition 1] it follows that for complex $O^{*}$-algebras our notion of bounded elements agrees with a similar notion in $[12,13]$.

Theorem 3.3 In the $O^{*}$-algebra $A$ of all bounded elements in an arbitrary real $O^{*}$-algebra E there exists a norm $\|\cdot\|$ with the following properties:

1) $\|\mathbf{I}\|=1$;

2) $\|x y\| \leq\|x\|\|y\|$ for all $x, y \in A$;

3) $\left\|x^{*}\right\|=\|x\|$ for all $x, y \in A$;

4) $\left\|x^{*} x\right\|=\|x\|^{2}$ for all $x, y \in A$;

5) $A_{h}$ is complete in the norm \| \|.

Proof Consider the hermitian part $A_{h}$ of $A$. For a hermitian $x \in E_{h}$ boundedness means that $0 \leq x^{2} \leq \lambda \mathbf{I}$ for some $\lambda \geq 0$, that is $-\sqrt{\lambda} \mathbf{I} \leq x \leq \sqrt{\lambda} \mathbf{I}$, i.e., $x$ is bounded in the $O J$-algebra $E_{h}$. Therefore (see [13, Ch. III, $\S 3$, Theorem 1]) $A_{h}$ is an order-unit space with respect to the norm

$$
\|a\|_{1}=\inf \{\lambda>0:-\lambda \mathbf{I} \leq a \leq \lambda \mathbf{I}\} .
$$

Moreover $\left(A_{h},\|\cdot\|_{1}\right)$ is a monotone complete $J B$-algebra (Jordan Banach algebra) - i.e., $O J B$-algebra in terms of $[3,13]$.

Now let $x \in A, x \neq 0$ be an arbitrary element and put $y=-x^{*} x$. The positive cone $K=\left\{a \in A_{h}: a \geq 0\right\}$ is closed in the $J B$-algebra $A_{h}$ with respect to the norm $\|\cdot\|_{1}$ (see [13, Ch. III, §2, Theorem 1]). From the axiom (4) of real $O^{*}$-algebras one has $y \neq 0$, and from Hahn-Banach's theorem we have that there exists a continuous linear functional $\varphi$ on $A_{h}$, which is positive on $K$, but $\varphi(y)<0$. Let us extend $\varphi$ from $A_{h}$ to the whole $A$ putting $\varphi(a)=\varphi\left(\frac{1}{2}\left(a+a^{*}\right)\right), a \in A$, i.e., $\varphi$ is zero on skewhermitian elements of $A$ (i.e., $\varphi(b)=0$ for $b^{*}=-b$ ). It is clear that $\varphi$ is a linear functional on $A, \varphi\left(a a^{*}\right) \geq 0$ for any $a \in A$ and $\varphi\left(x^{*} x\right)=-\varphi(y)>0$ for $x$ given above.

For the functional $\varphi$ on $A$ we have the following Schwarz inequality

$$
\varphi\left(a^{*} b\right)^{2} \leq \varphi\left(a^{*} a\right) \varphi\left(b^{*} b\right), \quad a, b \in A .
$$


Indeed, since $a^{*} b-b^{*} a$ is skew-hermitian, one has $\varphi\left(a^{*} b-b^{*} a\right)=0$, i.e., $\varphi\left(a^{*} b\right)=\varphi\left(b^{*} a\right)$. Further $\varphi\left((a-\lambda b)^{*}(a-\lambda b)\right) \geq 0$ for an arbitrary $\lambda \in \mathbb{R}$.

Therefore

$$
\varphi\left(a^{*} a\right)-\lambda \varphi\left(a^{*} b+b^{*} a\right)+\lambda^{2} \varphi\left(b^{*} b\right) \geq 0,
$$

i.e.

$$
\varphi\left(a^{*} a\right)-2 \lambda \varphi\left(a^{*} b\right)+\lambda^{2} \varphi\left(b^{*} b\right) \geq 0 .
$$

Thus the discriminant of this quadratic form is negative, i.e.,

$$
4 \varphi\left(a^{*} b\right)^{2}-4 \varphi\left(a^{*} a\right) \varphi\left(b^{*} b\right) \leq 0,
$$

i.e.,

$$
\varphi\left(a^{*} b\right)^{2} \leq \varphi\left(a^{*} a\right) \varphi\left(b^{*} b\right) .
$$

In particular putting $a=\mathbf{I}, b=x^{*} x$ one obtains

$$
\varphi\left(x^{*} x\right)^{2} \leq \varphi(\mathbf{I}) \varphi\left(x^{*} x x^{*} x\right) .
$$

Since $\varphi\left(x^{*} x\right)>0$ we have that $\varphi(\mathbf{I})>0$, and therefore without loss of generality we may assume that $\varphi(\mathbf{I})=1$, i.e., $\varphi$ is a state on the $*$-algebra $A$. Now let us construct a representation of $A$ by operators on a real Hilbert space associated with the state $\varphi$ (a real GNS-construction). Consider on $A$ the bilinear form $(a, b)=\varphi\left(b^{*} a\right), a, b \in A$.

As it was showed above $\varphi\left(a^{*} b\right)=\varphi\left(b^{*} a\right)$, i.e., $(a, b)=(b, a)$ for any $a, b \in A$, i.e., this bilinear form is symmetric. Put $J=\left\{a \in A:(a, a)=\varphi\left(a^{*} a\right)=0\right\}$ and let us prove that $J$ is a left ideal in $A$. If $a \in J, b \in A$ then by the above Schwarz inequality $\varphi(b a)^{2} \leq \varphi\left(b b^{*}\right) \varphi\left(a a^{*}\right)=0$. Therefore given any $a_{1}, a_{2} \in J$ we have $\varphi\left(\left(a_{1}+a_{2}\right)^{*}\left(a_{1}+\right.\right.$ $\left.\left.a_{2}\right)\right)=\varphi\left(a_{1}^{*} a_{1}\right)+\varphi\left(a_{1}^{*} a_{2}\right)+\varphi\left(a_{2}^{*} a_{1}\right)+\varphi\left(a_{2}^{*} a_{1}\right)=0$, i.e., $a_{1}+a_{2} \in J$. If $\lambda \in \mathbb{R}, a \in J$ then it is clear that $\lambda a \in J$, i.e., $J$ is a linear subspace in $A$. Moreover if $a \in J, b \in A$ then from above we have

$$
\varphi\left((b a)^{*}(b a)\right)=\varphi\left(a^{*} b^{*} b a\right)=\varphi\left(\left(a^{*} b^{*} b\right) a\right)=0,
$$

i.e., $b a \in J$, which means that $J$ is a left ideal in $A$.

Denote by $D_{\varphi}$ the factor space $A / J$. If $\xi, \eta \in D_{\varphi}$ and $a, b$ are representatives of these classes respectively, then putting

$$
(\xi, \eta)=(a, b)=\varphi\left(b^{*} a\right)
$$

we obtain a well-defined inner product on $D_{\varphi}$, i.e., $D_{\varphi}$ becomes a pre-Hilbert space. Taking the completion of $D_{\varphi}$ by the norm $\|\xi\|_{\varphi}=\sqrt{(\xi, \xi)}=\sqrt{\varphi\left(a^{*} a\right)}, a \in \xi$, we obtain a Hilbert space $H_{\varphi}$.

Further in the standard way we construct a regular representation $\Pi_{\varphi}$ of the algebra $A$ on this Hilbert space such that $\Pi_{\varphi}(x) \neq 0$ for the fixed element $x \neq 0$, $x \in A$, such that $\varphi\left(x^{*} x\right)>0$. Now if $\Im$ is the family of all states on $A$, then $\Pi=\bigoplus_{\varphi \in \Im} \Pi_{\varphi}$ gives a faithful representation of $A$ in the Hilbert space $H=\bigoplus_{\varphi \in \Im} H_{\varphi}$. Similarly to the complex case $[13, \mathrm{Ch}$ IV] one can define a new norm on $A$ putting $\|x\|=\|\Pi(x)\|$, where the latter norm denotes the operator norm in $B(H)$, and show that $\|x\|_{1}=\|x\|$ for all $x \in A_{h}$. Therefore $A_{h}$ is complete in the norm \| $\|$. The proof is complete. 
Remark Unlike the complex case the whole $O^{*}$-algebra $A$ of bounded elements may not be complete as the following example shows.

Example 6 Put $E=s+i c_{f}$, where $s$ is the algebra of all real sequences, $c_{f}$-the algebra of all real sequences with a finite number of non zero terms. Then $E$ is a real $*$-algebra: indeed for $f_{1}+i g_{1}, f_{2}+i g_{2} \in E, f_{1}, f_{2} \in s, g_{1}, g_{2} \in c_{f}$, we have

$$
\left(f_{1}+i g_{1}\right)+\left(f_{2}+i g_{2}\right)=\left(f_{1}+f_{2}\right)+i\left(g_{1}+g_{2}\right) \in E
$$

and

$$
\left(f_{1}+i g_{1}\right) \cdot\left(f_{2}+i g_{2}\right)=\left(f_{1} f_{2}-g_{1} g_{2}\right)+i\left(f_{1} g_{2}+g_{1} f_{2}\right) \in E
$$

because $f_{1}+f_{2}, f_{1} f_{2}-g_{1} g_{2} \in s, g_{1}+g_{2}, f_{1} g_{2}+g_{1} f_{2} \in c_{f}$. Since $E_{h}=s$ the axioms of real $O^{*}$-algebras are obviously satisfied by $E$. The $O^{*}$-subalgebra of bounded elements of $E$ coincides with the algebra $A=m+i c_{f}$, where $m$ is the algebra of all bounded real sequences. The algebra $A$ is not complete in sup-norm. Indeed if we consider the sequence $x_{n}=\left(1, \frac{1}{2}, \frac{1}{3}, \ldots, \frac{1}{n}, 0,0, \ldots\right) \in c_{f}$ then the sequence $\left\{0+i x_{n}\right\}$ is fundamental because $\left\|x_{n}-x_{m}\right\|=\frac{1}{m} \rightarrow 0, n>m \rightarrow \infty$. But $x_{n} \rightarrow\left(1, \frac{1}{2}, \frac{1}{3}, \ldots, \frac{1}{m}, \ldots\right) \notin c_{f}$, i.e., $\left\{0+i x_{n}\right\}$ does not converge in $A=m+i c_{f}$. Therefore $A$ is not complete.

\section{Commutation in Real O*-algebras}

In this section we shall consider more deeply commutation properties in terms of symmetrizes (i.e., Jordan) products.

Proposition 4.1 Let $E$ be a real $O^{*}$-algebra, $a, x \in E$ and suppose that $a$ is hermitian (i.e., $a^{*}=a$ ) and $x$ is skew-hermitian (i.e., $x^{*}=-x$ ). And let $a=\int_{-\infty}^{+\infty} \lambda d e_{\lambda}$ be the spectral resolution of the hermitian element $a$. Then $[a, x]=0$ if and only if $\left[e_{\lambda}, x\right]=0$ for all $e_{\lambda}, \lambda \in \mathbb{R}$ (the spectral projections of $a$ ).

Proof It is known that the spectral projections $\left\{e_{\lambda}\right\}$ of any hermitian element $a \in$ $E$, belong to each maximal abelian subalgebra of $E$ which contains $a$ [13, Ch.III]. Suppose that $[a, x]=0$. Then since $a=a^{*}$ and $x=-x^{*}$, we have that all elements $a, a^{*}, x, x^{*}$ pair-wise commute, i.e., the *-subalgebra $A(a, x)$ generated by $a$ and $x$ is abelian. Consider a maximal abelian *-subalgebra $\tilde{E}$ containing $A(a, x)$. Then $e_{\lambda} \in \tilde{E}$ for all $\lambda \in \mathbb{R}$ and therefore $\left[e_{\lambda}, x\right]=0$ for all $\lambda \in \mathbb{R}$. Conversely, if $\left[e_{\lambda}, x\right]=0$ for all $\lambda \in \mathbb{R}$ then as above there exists a maximal abelian ${ }^{*}$-subalgebra $\tilde{E}$ containing $x$ and all $e_{l}, \lambda \in \mathbb{R}$, because $\left[e_{\lambda}, e_{\mu}\right]=0$ for all $\lambda, \mu \in \mathbb{R}$. But then $a \in \tilde{E}$, therefore $[a, x]=0$. The proof is complete.

The following corollary strengthens Proposition 2.1.

Corollary 4.2 Let $E$ be a real $O^{*}$-algebra, $E_{h}$ its hermitian part. For $a, b \in E_{h}$ the following conditions are equivalent

a) $\{a, c, b\}=0$ for all $c \in E_{h}$;

B) $\{a, a, b\}=0$; 
$\left.\beta^{\prime}\right) \quad\{a, b, b\}=0$

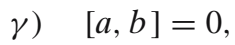

where $\{a, c, b\}=(a \circ c) \circ b-a \circ(c \circ b)$ is the associator in the Jordan product $x \circ$ $y=\frac{1}{2}(x y+y x)$, and $[a, b]=a b-b a$ is the commutator in the associative product.

Proof In view of the identity $\{a, c, b\}=\frac{1}{4}[c,[a, b]]$ the implication $\left.\gamma\right) \Rightarrow \alpha$ ) is obvious. Also it is clear that $\alpha) \Rightarrow \beta$ ) and $\alpha) \Rightarrow \beta^{\prime}$ ). Therefore it is sufficient to prove that $\beta) \Rightarrow \gamma$ ), i.e., $[a,[a, b]]=0 \Rightarrow[a, b]=0$.

First suppose that $a$ is a projection, i.e. $a=a^{2}=a^{*}$. Then $[a,[a, b]]=a b-a b a-$ $a b a+b a=a b-2 a b a+b a=0$. Multiplying this equality by $a$ from the right side we obtain $a b a-2 a b a+b a=0$, i.e., $b a=a b a$.

Similarly $a b=a b a$, i.e., $a b=b a$, so $[a, b]=0$ and the assertion is proved in this case.

Turning to the general case put $d=[a, b]$. Then since $a, b \in E_{h}$, we have $d^{*}=-d$ and, by the assumption, $[a, d]=0$. If $a=\int_{-\infty}^{+\infty} \lambda d e_{\lambda}$ is the spectral resolution of $a$ the Proposition 4.1 implies that $\left[e_{\lambda}, d\right]=0$ for all $\lambda \in \mathbb{R}$. Therefore $\left[e_{\lambda},[a, b]\right]=0$ for any $\lambda \in \mathbb{R}$.

From the Jacobi identity we have

$$
\left[[a, b], e_{\lambda}\right]+\left[\left[b, e_{\lambda}\right], a\right]+\left[\left[e_{\lambda}, a\right], b\right]=0 .
$$

Since $\left[[a, b], e_{\lambda}\right]=0$ and $\left[e_{\lambda}, a\right]=0$ it follows that $\left[\left[b, e_{\lambda}\right], a\right]=0$. But the element $\left[b, e_{\lambda}\right]$ is also skew-hermitian. Applying Proposition 4.1. once more we have $\left[\left[b, e_{\lambda}\right], e_{\lambda}\right]=0$. But $e_{\lambda}$ is a projection and from above we obtain that $\left[b, e_{\lambda}\right]=0$ for all $\lambda \in \mathbb{R}$. Again Proposition 4.1 implies that $[b, a]=0$. The proof is complete.

The set $Z_{E}=\{x \in E: \quad x y=y x, \forall y \in E\}$ is called the center of the real $O^{*}$-algebra $E$. It is clear that elements of the form $\lambda \mathbf{I}, \lambda \in \mathbb{R}$, are in the center of $E$.

Definition 4 A real $O^{*}$-algebra $E$ is said to be a real $O^{*}$-factor, if $Z_{E}=\{\lambda \mathbf{I}, \lambda \in \mathbb{R}\}$, i.e., the center of $E$ consists of real multiples of the identity element.

Note that a complex $O^{*}$-algebra $F$ is called $O^{*}$-factor if $Z_{F}=\{\lambda \mathbf{I}, \lambda \in \mathbb{C}\}$. Therefore $O^{*}$-factors are real $O^{*}$-algebras, but not real $O^{*}$-factors!

Corollary 4.3 In a real $O^{*}$-algebra $E$ non zero central elements can not be represented as a commutator of hermitian elements. In particular there is no Heisenberg representation of canonical commutation relations by hermitian elements of complex $O^{*}$-algebras (i.e., there is no $a, b \in E_{h}$, such that $[a, b]=i \mathbf{I}$ ).

Proof If $[a, b] \in Z_{E}$, then in particular $[[a, b], a]=0$, therefore $[a, b]=0$.

In order to obtain a criterion for an element of a real $O^{*}$-algebra to be central we need the following technical result. Recall that an element $u$ of a real $O^{*}$-algebra is called a unitary element, if $u^{*} u=u u^{*}=\mathbf{I}$.

Proposition 4.4 In a real $O^{*}$-algebra each bounded skew-hermitian element is a linear combination of two unitary elements. 
Proof Let $x^{*}=-x$ be a bounded element, i.e., $0 \leq-x^{2}=x^{*} x \leq \lambda \mathbf{I}$ for some $\lambda \in \mathbb{R}$, $\lambda>0$. Passing if necessary to the element $\lambda^{-1} x$, we may suppose that $x^{*} x=-x^{2} \leq \mathbf{I}$. Then $\mathbf{I}+x^{2} \geq 0$ and therefore there exists a positive square root $h=\sqrt{\mathbf{I}+x^{2}}$, commuting with $x$ (in fact $h=\int_{0}^{1} \sqrt{\lambda} d e_{\lambda}$, where $\mathbf{I}+x^{2}=\int_{0}^{1} \lambda d e_{\lambda}$ is the spectral resolution of $\left.\mathbf{I}+x^{2}\right)$.

Put $u_{1}=x+h, u_{2}=x-h$, then it is clear that $x=\frac{1}{2}\left(u_{1}+u_{2}\right)$ and

$$
u_{1}^{*} u_{1}=\left(x^{*}+h\right)(x+h)=(h-x)(h+x)=h^{2}-x^{2}=\mathbf{I}+x^{2}-x^{2}=\mathbf{I},
$$

and similarly $u_{1} u_{1}^{*}=\mathbf{I}$.

Also $u_{2}^{*} u_{2}=\left(x^{*}-h\right)(x-h)=-(x+h)(x-h)=-x^{2}+h^{2}=-x^{2}+\mathbf{I}+x^{2}=\mathbf{I}$ and similarly $u_{2} u_{2}^{*}=\mathbf{I}$, i.e., $u_{1}$ and $u_{2}$ are unitaries. The proof is complete.

Proposition 4.5 An element $x$ in a real $O^{*}$-algebra $E$ belongs to the center $Z_{E}$ if and only if $u^{*} x u=x$ for any unitary $u \in E$.

Proof If $x \in Z_{E}$ then it is clear that $u^{*} x u=x$ for any unitary $u \in E$.

Conversely, suppose that $u^{*} x u=x$ for each unitary $u \in E$. Applying the involution to this equality we obtain $u^{*} x^{*} u=x^{*}$ and thus

$$
u^{*}\left(x+x^{*}\right) u=x+x^{*}, u^{*}\left(x-x^{*}\right) u=x-x^{*}
$$

for any unitary $u \in E$. Consider the hermitian element $a=x+x^{*}$. If $e \in E$ is a projection then $\mathbf{I}-2 e$ is a unitary, because $(\mathbf{I}-2 e)^{*}(\mathbf{I}-2 e)=\mathbf{I}-2 e-2 e+4 e=\mathbf{I}$. Therefore $(\mathbf{I}-2 e) a(\mathbf{I}-2 e)=a$, i.e., $a-2 e a-2 a e+4 e a e=a$, i.e., $2 e a e=e a+a e$. Multiplying this by $e$ from the right, one has $2 e a e=e a e+a e$, i.e., $a e=e a e$. Similarly $e a e=e a$, i.e., $e a=a e$. Therefore $a$ commutes with every projection $e \in E$ and by the spectral theorem $a$ commutes with each hermitian element in $E$. Now consider a skew-hermitian element $y \in E$ and take the hermitian element $\mathbf{I}+y^{*} y=$ $\mathbf{I}-y^{2} \geq \mathbf{I}$. Then it is clear that $\mathbf{I}-y^{2}$ is invertible and its inverse $\left(\mathbf{I}-y^{2}\right)^{-1}$ belongs to any maximal abelian $*$-subalgebra, containing $\mathbf{I}-y^{2}$. From the above we have that both $\left(\mathbf{I}-y^{2}\right)$ and $\left(\mathbf{I}-y^{2}\right)^{-1}$ commute with $a$. Put $t=y\left(\mathbf{I}-y^{2}\right)^{-1}$. This element is skew-hermitian and

$$
t^{*} t=-t^{2}=\frac{-y^{2}}{\mathbf{I}-y^{2}} \leq \frac{\mathbf{I}-y^{2}}{\mathbf{I}-y^{2}}=\mathbf{I},
$$

i.e., $t$ is bounded. By Proposition $4.4 t$ is a linear combination of two unitaries. Therefore $a$ commutes with $t$ (since $u^{*} a u=a$ multiplying by $u$ from the left side we obtain $a u=u a)$ i.e.,

$$
a y\left(\mathbf{I}-y^{2}\right)^{-1}=y\left(\mathbf{I}-y^{2}\right)^{-1} a .
$$

Multiplying this from the right side by the element $\mathbf{I}+y^{2}$ and noting that $\mathbf{I}-y^{2}$ commutes with $a$, we have

$$
a y=y a .
$$

Thus the element $a=x^{*}+x$ commutes with any skew-hermitian $y \in E$. But any element in $E$ is the sum of a hermitian and a skew-hermitian elements. Therefore $a \in Z_{E}$. Similarly applying the Proposition 4.1 we prove that the skew-hermitian element $x-x^{*}$ is in $Z_{E}$. Therefore $x \in Z_{E}$. The proof is complete. 
We conclude this section by a description of so called discrete real $O^{*}$-factors. Let $E$ be a real $O^{*}$-factor, i.e., $Z_{E}=\{\lambda \mathbf{I}, \lambda \in \mathbb{R}$. $\}$

Definition $5 E$ is said to be discrete or type $I$, if its lattice of projections is atomic, i.e., given any projection $e \in E$ there exists an atom (minimal projection) $p$ such $p \leq e$.

Theorem 4.6 Let $E$ be a real $O^{*}$-factor. If $E$ contains at least one atom, then $E$ is a discrete real $O^{*}$-factor and isomorphic to the algebra $B(H)$ of all bounded linear operators on a real or quaternionic Hilbert space $H$. In particular all elements are bounded.

Proof Consider the $O^{*}$ subalgebra $B$ of all bounded elements of $E$. Then

$$
B_{h}=\left\{x \in B: x^{*}=x\right\}
$$

is an $O J B$-factor in the sense of [13, Ch.III]. If $p$ is an atom in $E$ (and thus in $B_{h}$ ) then $p B_{h} p$ contains only two projections 0 or $p$, i.e., $p B_{h} p \cong \mathbb{R} p$ (real multiples of $p$ ). Thus given any $x \in B_{h}$, we have $p x p=\lambda p$ for an appropriate real $\lambda=\lambda(x)$, i.e., $\operatorname{pxp}=\lambda_{p}(x) p$. It is easy to see that the functional $\lambda_{p}: B_{h} \rightarrow \mathbb{R}$ is a state on $B_{h}$ and it is normal, since the map $x \rightarrow p x p$ is normal. Therefore the $O J B$-factor $B_{h}$ admits a normal state $\lambda_{p}$. By [13, Ch.III, $\$ 9$, Corollary 1 of Theorem 3] it follows that $B_{h}$ is a $J W$-factor - a weakly closed Jordan algebra of self-adjoint bounded operators on a complex Hilbert space $[3,5,16]$, with a trivial center. Further since $B_{h}$ contains a minimal projection $p$, it is a $J W$-factor of type $I$, and therefore by [5, Ch.II, Theorem 2.1] (see also $[3,16]$ ), $B_{h}$ is isomorphic to the algebra $B(H)_{S A}$ of all bounded self-adjoint operators on a real $\left(H=H_{\mathbb{R}}\right)$, complex $\left(H=H_{\mathbb{C}}\right)$ or quaternionic $\left(H=H_{\mathbb{Q}}\right)$ Hilbert space. But then by [13, Ch.III, §4, Theorem 4.2] $E_{h}$ can be embedded into the $O J$-algebra $S\left(B(H)_{S A}\right.$ ) of all locally measurable operators affiliated with $B(H)_{S A}$. For $J W$-factors $A$ of type $I$, it is known that $S(A)=A$, therefore $E_{h}=B_{h}$ i.e., all hermitian elements of the real $O^{*}$-factor $E$ are bounded. From the definition it follows that all elements of $E$ are also bounded, i.e., $E=B$. Therefore we have proved that $E=B$ and $E_{h} \cong B(H)_{S A}$, and by $G N S$-construction for the $O^{*}$-algebras of bounded elements (see Theorem 3.3) $E$ can be embedded into $B(H)$ i.e., $E \subset B(H)$. If $H=H_{\mathbb{C}}$ then $E=E_{h}+E_{k}=$ $B\left(H_{\mathbb{C}}\right)_{S A}+i B\left(H_{\mathbb{C}}\right)_{S A}=B\left(H_{\mathbb{C}}\right),\left(E_{k}\right.$ denotes the skew-hermitian part of $\left.E\right)$. But $B\left(H_{\mathbb{C}}\right)$ is not a real $O^{*}$-factor, therefore the case $H=H_{\mathbb{C}}$ is impossible. For $H=H_{\mathbb{R}}$ or $H_{\mathbb{Q}}$ any skew-hermitian operator from $B(H)$ is a finite sum of commutators of hermitian elements from $B(H)_{S A}=E_{h}$ [9] and thus $B(H)_{k} \subset E_{k}$. Therefore $B(H)=$ $B(H)_{S A} \oplus B(H)_{k} \subset E_{h}+E_{k}=E$, i.e., $E=B(H)$, where $H=H_{\mathbb{R}}$ or $H_{\mathbb{Q}}$. The proof is complete.

Corollary 4.7 (Analogue of Frobenius' theorem). Let a real $O^{*}$-algebra $E$ have only two projections $\mathbf{0}$ and $\mathbf{I}$. Then $E$ is $*$-isomorphic to $\mathbb{R}, \mathbb{C}$, or $\mathbb{Q}$.

Proof One can obtain the proof from the theorem above and from the description of complex discrete $O^{*}$-factors [13]. But here we suggest a straightforward proof. By the spectral theorem we have that any hermitian element $a \in E_{h}$ is a real multiple of $\mathbf{I}$, i.e., $a=\lambda \mathbf{I}, \lambda \in \mathbb{R}$. Let us prove that $E$ is a division algebra, i.e., any non-zero 
element is invertible in $E$. Let $x \in E, x \neq 0$. By the axiom 4) of real $O^{*}$-algebras $x^{*} x \in E_{h}, x^{*} x \neq 0$, i.e., $x^{*} x=\lambda \mathbf{I}, \lambda \in \mathbb{R}, \lambda \neq 0$. Similarly $x x^{*}=\mu \mathbf{I}, \mu \in \mathbb{R}, \mu \neq 0$. Multiplying this from the right side by $x$, we obtain $x x^{*} x=\mu x$, i.e., $x(\lambda \mathbf{I})=\mu x$, i.e., $\lambda x=\mu x$. Since $x \neq 0$ one has $\lambda=\mu$, i.e., $x^{*} x=x x^{*}=\lambda \mathbf{I}$, i.e., $\lambda^{-1} x^{*}$ is the inverse of $x$, i.e., $E$ is a division algebra over $\mathbb{R}$. By the classical Frobenius' theorem $E$ is isomorphic to either $\mathbb{R}, \mathbb{C}$ or $\mathbb{Q}$. The proof is complete.

\section{O*-algebras of Locally Measurable Operators and Representations of Real O*-algebras}

It is known that in the complex case under some natural conditions an abstract $O^{*}$-algebra can be embedded into the concrete $O^{*}$-algebra of all locally measurable operators affiliated with a von Neumann algebra (see e.g., [13, Ch.IV]). In this section we are going to obtain a similar result for real $\mathrm{O}^{*}$-algebras. For this sake it is necessary to define the algebras of measurable and locally measurable operators affiliated with a real von Neumann algebra $[8,11]$. It is somewhat routine if we follow step by step the complex case. Therefore we shall choose a shorter way and apply the method of complexification of given real algebras.

Recall $[5,8]$ that a real *-subalgebra $\Re$ of linear bounded operators on a complex Hilbert space $H$ (i.e., $\Re \subset B(H)$ ) is called a real von Neumann algebra, if it is closed in the weak operator topology, contains the identity operator $\mathbf{I}$ and $\Re \bigcap i \Re=\{0\}$. The smallest (complex) von Neumann algebra generated by the real von Neumann algebra $\Re$ coincides with its complexification: $\mathcal{U}(\Re)=\Re+i \Re$.

In this case $\Re$ generates the canonical involutive *-anti-automorphism of $\mathcal{U}(\Re)$ as

$$
\alpha_{\Re}(x+i y)=x^{*}+i y^{*}
$$

for $x+i y \in \mathcal{U}(\Re)=\Re+i \Re, x, y \in \Re$. Moreover $\Re=\left\{x \in \mathcal{U}(\Re): \alpha_{\Re}(x)=x^{*}\right\}$ (cf. Section 3). Conversely if $\alpha$ is an involutive *-anti-automorphism of a von Neumann algebra $\mathcal{U}$, then the set $\left\{x \in \mathcal{U}: \alpha(x)=x^{*}\right\}$ forms a real von Neumann algebra, generating $\mathcal{U}$.

We recall some definitions and notions from non commutative integration theory $[15,17]$. Let $H$ be a complex Hilbert space, and let $T$ denote a closed linear operator (not necessary bounded) with a dense domain $D(T)$. An operator $T$ is said to be positive (denoted as $T \geq 0$ ) if $(T \xi, \xi) \geq 0$ for all $\xi \in D(T)$. It is known that $T^{*} T \geq 0$ for any closed $T$. We say that $T$ commutes with the bounded operator $S \in B(H)$, if $S T \subset T S, S(D(T)) \subset D(T)$ and $S T \xi=T S \xi$ for all $\xi \in D(T)$.

Given any self-adjoint operator $T$ there exists a spectral family of projections $E(\lambda),-\infty<\lambda<+\infty$ such that $T \xi=\int_{-\infty}^{+\infty} \lambda d E(\lambda)$ for all $\xi \in D(T)$ (Spectral Theorem).

An operator $T$ in $H$ is said to be affiliated with a von Neumann algebra $M$ (denoted $T \eta M)$, if it commutes with each unitary operator $U$ from the commutant $M^{\prime}$ of $M$ in $B(H)$. It is clear that if $T$ is bounded (i.e., $T \in B(H)$ ) then $T \eta M$ and only if $T \in M$, since in this case $T \in M^{\prime \prime}=M$ (bicommutant theorem). Further, if $T$ is self-adjoint, then $T \eta M$ and only if $M$ contains all spectral projection $E(\lambda)$ of $T$. 
Definition 6 Let $T$ be a closed operator affiliated with $M$, i.e., $T \eta M$. $T$ is said to be measurable (with respect to $M$ ) if the projection $\mathbf{I}-E(\lambda)$ is finite in $M$ for some $\lambda>0$, where $\{E(\lambda)\}$ is the spectral family of the positive operator $|T|=\left(T^{*} T\right)^{\frac{1}{2}}$.

Measurable operators were studied by Segal in [15], where in particular he has proved that the set $C(M)$ of all measurable operators affiliated with $M$ forms a complex *-algebra with respect to natural algebraic operations.

Definition 7 An operator $T$ in $H$ is called locally measurable (with respect to $M$ ), if there is a sequence of central projections $\left\{P_{n}\right\}$ in $M$ increasing to $\mathbf{I}$ such that $P_{n} T$ is measurable for all $n=1,2, \ldots$.

The set $S(M)$ of locally measurable operators was considered by Yeadon [17], who has proved that $S(M)$ forms a monotone complete *-algebra. In the monograph [13] it is proved that $S(M)$ is a complex $O^{*}$-algebra and $C(M)$ is a solid $O^{*}$-subalgebra in $S(M)$ (i.e., $0 \leq X \leq Y \in C(M), X \in S(M)$ implies $x \in C(M)$ ). Moreover the $O^{*}$-subalgebra of bounded element both of $C(M)$ and $S(M)$ coincides with the von Neumann algebra $M$.

Now let $\Re$ be a real von Neumann algebra in $B(H), M=\Re+i \Re$ its enveloping von Neumann algebra, $\alpha$ - the corresponding involutive *-anti-automorphism of $M$, generated by $\Re$. A natural question arises:

Can the *anti-automorphism $\alpha$ be extended to $a^{*}$-anti-isomorphism of $C(M)$ or $S(M)$ ?

In order to give an answer to this question we need the following theorem which extends the Theorem 1 from [13, Ch.IV, §7] for *-anti-isomorphisms.

Theorem 5.1 Let $E, \bar{E}$ be complex $O^{*}$-algebras, $A, \bar{A}$-the $O C^{*}$-algebras of bounded elements of $E$ and $\bar{E}$ respectively. And suppose that $\Phi: A \rightarrow \bar{A} a^{*}$-antiisomorphism between $A$ and $\bar{A}$ satisfying the following condition:

(*) given any $a \in E_{h}$ with the spectral family of projections $\left\{e_{\lambda}\right\}$ the integral $\int_{-\infty}^{+\infty} \lambda d \Phi\left(e_{\lambda}\right)$ converges in $\bar{E}_{h}$.

Then $\Phi$ can be uniquely extended to $a^{*}$-anti-isomorphism $\bar{\Phi}$ of $E$ onto a solid $O^{*}$-subalgebra of $\bar{E}$.

Proof One can follow step by step the proof of Theorem 1 from [13, Ch. IV, Section 7] replacing the word "*-isomorphism" by the word "*-anti-isomorphism".

Corollary 5.2 Let $E$ be a complex $O^{*}$-algebra with the subalgebra $A$ of all bounded element. Suppose that $\alpha: A \rightarrow A$ is an involutive *-anti-automorphism of the $O C^{*}$-algebra $A$. Then $\alpha$ can be uniquely extended to an involutive *-antiautomorphism $\bar{\alpha}$ of the solid $O^{*}$-subalgebra

$$
E_{\alpha}=\left\{x \in E: \int_{0}^{+\infty} \lambda d \alpha\left(e_{\lambda}\right) \text { exists }\right\},
$$

where $e_{\lambda}$ is the spectral family of the element $|x|$.

Springer 
Now turning to the above question consider the $O^{*}$-algebras $C(M)$ and $S(M)$ and the involutive ${ }^{*}$-anti-automorphism $\alpha: M \rightarrow M$ generated by the real von Neumann algebra $\Re$.

Given an operator $T \geq 0$ in $H$ it is clear that $T \eta M$ if and only if its spectral projections $E(\lambda)$ are in $M$. But then $\alpha(E(\lambda))$ are also in $M$ and therefore $\int_{0}^{+\infty} \lambda d(E(\lambda)) \eta M$, i.e.,

$$
T=\int_{0}^{+\infty} \lambda d E(\lambda) \eta M \Leftrightarrow \bar{\alpha}(T)=\int_{0}^{+\infty} \lambda d \alpha(E(\lambda)) \eta M .
$$

Further a projection $e \in M$ is finite if and only if $\alpha(e)$ is finite in $M$. Therefore $T \in$ $C(M) \Leftrightarrow \bar{\alpha}(T)=\int_{0}^{+\infty} \lambda d \alpha(E(\lambda)) \in C(M)$.

Finally, if $\left\{P_{n}\right\}$ is an increasing sequence of central projections in $M$ with sup $P_{n}=\mathbf{I}$ and $P_{n} T \in C(M)$, then $\left\{\alpha\left(P_{n}\right)\right\}$ is also a sequence of central projections which increases to $\mathbf{I}$ and $\alpha\left(P_{n}\right) \bar{\alpha}(T)=\bar{\alpha}\left(P_{n} T\right) \in C(M)$. Therefore

$$
T \in S(M) \Leftrightarrow \bar{\alpha}(T)=\int_{0}^{+\infty} \lambda d \alpha(E(\lambda)) \in S(M) .
$$

The above implications show that in the cases of the complex $O^{*}$-algebras $C(M)$ and $S(M)$ the corresponding solid $O^{*}$-subalgebras $E_{\alpha}$ coincide with the whole $O^{*}$-algebras $C(M)$ and $S(M)$ respectively. Therefore we obtain the following

Theorem 5.3 Any involutive *-anti-automorphism $\alpha$ of the (complex) von Neumann algebra $M$ can be uniquely extended to the involutive ${ }^{*}$-anti-automorphism $\bar{\alpha}$ of the $O^{*}$-algebras $C(M)$ and $S(M)$.

Remark The assertion of the above theorem does not hold for arbitrary $O^{*}$-algebras with the bounded part $M$. Indeed, consider the abelian $O^{*}$-algebra $E=m \oplus s-$ the direct sum of the abelian $O^{*}$-algebras $m$ (of all bounded sequences of real numbers) and $s$ (of all sequences of real numbers). Then the ${ }^{*}$-subalgebra of bounded elements in $E$ coincides with the abelian von Neumann algebra $m \oplus m$. Consider the involutive *-anti-automorphism (which is simply a ${ }^{*}$-automorphism in view of the commutativity of $M) \alpha: M \rightarrow M$, defined by

$$
\alpha:(a, b) \rightarrow(b, a),(a, b) \in M=m \oplus m .
$$

Then it is clear that the $O^{*}$-subalgebra $E_{\alpha}$ from above coincides exactly with $M$, i.e., $\alpha$ can not be extended to any unbounded part of $E=m \oplus s$. This example can be extended to the non abelian cases, e.g., considering $O^{*}$-algebras of the form $E=M \oplus S(M)$.

Now let $\Re$ be a real von Neumann algebra. We are going to introduce the notions of measurable and locally measurable operators with respect to $\Re$. To this end consider the complexification $M=\Re+i \Re$ of the given real von Neumann algebra $\Re$ and the corresponding involutive *-anti-automorphism $\alpha=\alpha_{\Re}$ of $M$, i.e., $\Re=\{x \in$ $\left.M: \alpha(x)=x^{*}\right\}$. 
Applying Theorem 5.3 let us extend $\alpha$ to an involutive ${ }^{*}$-anti-automorphism $\bar{\alpha}: S(M) \rightarrow S(M)$, which restricted to $C(M)$ gives an involutive *-anti-automorphism of $C(M)$.

Consider the real ${ }^{*}$-subalgebras

$$
S(\Re)=\left\{x \in S(M): \bar{\alpha}(x)=x^{*}\right\} \text { in } S(M)
$$

and

$$
C(\Re)=\left\{x \in C(M): \bar{\alpha}(x)=x^{*}\right\} \text { in } C(M) .
$$

It is clear that

$$
S(M)=S(\Re)+i S(\Re) \text { and } C(M)=C(\Re)+i C(\Re) .
$$

Since $S(M)$ and $C(M)$ are (complex) $O^{*}$-algebras, Theorem 2.3 from Section 2 implies that both $S(\Re)$ and $C(\Re)$ are real $O^{*}$-algebras, with respect to the partial order induced from $S(M)$.

Definition 8 Operators from $C(\Re)$ (resp. from $S(\Re)$ ) are called measurable (resp. locally measurable) operators affiliated with the real von Neumann algebra $\Re$.

Thus we have the following result

Theorem 5.4 The algebras $S(\Re)$ of all locally measurable operators and $C(\Re)$ of all measurable operators affiliated with the real von Neumann algebra $\Re$ are real $O^{*}$-algebras and the ${ }^{*}$-subalgebra of all bounded elements in both $S(\Re)$ and in $C(\Re)$ ) coincides with $\Re$.

Now we are in position to prove the main result of this paper - a representation theorem for abstract real $O^{*}$-algebras.

Theorem 5.5 Let $E$ be a real $O^{*}$-algebra such that its complexification $F=E+i E$ is a (complex) $O^{*}$-algebra. Suppose also that the real ${ }^{*}$-subalgebra $B_{E}$ of all bounded elements in E possesses a separating set of normal states. Then $E$ is ${ }^{*}$-isomorphic to a solid $O^{*}$-subalgebra of the real $O^{*}$-algebra $S(\Re)$ of all locally measurable operators affiliated with an appropriate real von Neumann algebra $\Re$.

Proof Consider the involutive *-anti-automorphism $\alpha: a+i b \rightarrow a^{*}+i b^{*}, a, b \in E$, of the complex $O^{*}$-algebra $F=E+i E$. Then it is clear that the ${ }^{*}$-subalgebra $B_{F}$ of all bounded elements of the $O^{*}$-algebra $F$ coincides with the complexification of $B_{E}$, i.e., $B_{F}=B_{E}+i B_{E}$ and $B_{E}=\left\{x \in B_{F}: \alpha(x)=x^{*}\right\}$. Let us prove that the $O C^{*}$-algebra $B_{F}$ has a separating family of normal states. Let $\rho$ be a normal state on the real *-algebra $B_{E}$. For $x \in B_{F}, x=x^{*}$, put

$$
\bar{\rho}(x)=\rho\left(\frac{x+\alpha(x)}{2}\right) ;
$$

since $x+\alpha(x) \in B_{F}, \bar{\rho}$ is well defined.

It is clear that $\bar{\rho}$ is a real linear functional on $\left(B_{F}\right)_{h}$, and if $x \geq 0$ then $\bar{\rho}(x) \geq 0$. Therefore by the complex linearity $\bar{\rho}$ can be uniquely extended to a state on 基 Springer 
the whole $B_{F}$. Moreover if $x_{\lambda} \in\left(B_{F}\right)_{h}, x_{\lambda} \searrow 0$, then $x_{\lambda}+\alpha\left(x_{\lambda}\right) \searrow 0$ and by the normality of $\rho$ we have

$$
\bar{\rho}\left(x_{\lambda}\right)=\rho\left(\frac{x_{\lambda}+\alpha\left(x_{\lambda}\right)}{2}\right) \rightarrow 0
$$

so $\bar{\rho}$ is a normal state on $B_{F}$.

Thus given any normal state on the real $O^{*}$-algebra $B_{E}$ there is a corresponding normal state $\bar{\rho}$ on the $O C^{*}$-algebra $B_{F}$ (note that $\bar{\rho}$ is not necessarily the extension of $\rho$ ). If $x \in B_{F}, x \geq 0$, and $\bar{\rho}(x)=0$ for all normal states $\rho$ on $B_{E}$, then in view of the positivity of $x$ and $\alpha(x)$ this implies that $x+\alpha(x)=0$ and hence $x=\alpha(x)=0$. Therefore $\{\bar{\rho}\}$ gives a separating family of normal states on the $O C^{*}$-algebra $B_{F}$. By [13, Ch.IV, §7] the $O C^{*}$-algebra $B_{F}$ is *-isomorphic to a (complex) von Neumann algebra $M$, and $F$ is *-isomorphic to a solid $O^{*}$-subalgebra of the $O^{*}$-algebra $S(M)$ of all locally measurable operators affiliated with $M$. Now (identifying $B_{F}$ with $M$ ) if we put

$$
\Re=\left\{x \in M: \alpha(x)=x^{*}\right\}
$$

then we obtain a real von Neumann algebra $\Re$, which is *-isomorphic to $B_{E}$. Therefore the real $O^{*}$-algebra

$$
E=\left\{x \in F: \alpha(x)=x^{*}\right\}
$$

is *-isomorphic to a solid real $O^{*}$-subalgebra of the real $O^{*}$-algebra

$$
S(\Re)=\left\{x \in S(M): \bar{\alpha}(x)=x^{*}\right\}
$$

where $\bar{\alpha}$ is the unique extension of $\alpha$ to an involutive *anti-automorphism of $S(M)$ (see Theorem 5.3). The proof is complete.

Remark The additional condition that $F=E+i E$ is an $O^{*}$-algebra is crucial. As we can see from the Example 6 in Section 3 in general the ${ }^{*}$-subalgebra $B_{E}$ of all bounded elements in a real $O^{*}$-algebra $E$ may be not complete (i.e., $B_{E}$ is not even a real $C^{*}$-algebra) though it has a separating family of normal states. Therefore $B_{F}$ is not a real von Neumann algebra, so $E$ can not be a solid real $O^{*}$-subalgebra in the real $O^{*}$-algebra of the form $S(\Re)$ for some real von Neumann algebra $\Re$, because in this case $B_{F} \cong \Re-$ a contradiction.

Acknowledgements The second and third named authors would like to acknowledge the hospitality of the "Institut für Angewandte Mathematik", Universität Bonn (Germany). This work is supported in part by the DFG 436 USB $113 / 4$ project (Germany).

\section{References}

1. Albeverio, S., Ayupov, Sh.A., Abduvaitov, A.H.: On real $A W^{*}$-algebras. Methods Funct. Anal. Topology 11(2), 99-112 (2005)

2. Albeverio, S., Ayupov, Sh.A., Abduvaitov, A.H.: On coincidence of types of a real $A W^{*}$-algebra and its complexification. Izv. Ross. Akad. Nauk 68(5), 3-12 (2004)

3. Alfsen, E.M., Hanche-Olsen, H., Shultz, F.W.: State spaces of $C^{*}$-algebras. Acta Math. 144(3,4), 267-305 (1980)

4. Antoine, J.-P., Inoue, A., Trapani, C.: Partial *-algebras and Their Operator Realizations. Kluwer, Dordrecht, The Netherlands (2002) 
5. Ayupov, Sh.A.: Classification and Representation of Ordered Jordan Algebras (Monograph). Fan, Tashkent, Uzbekistan (1986)

6. Ayupov, Sh.A.: Real $A W^{*}$-algebras of type I. Funct. Anal. Appl. 38(3), 79-81 (2004)

7. Ayupov, Sh.A.: On $W^{*}$-embedding of real $A W^{*}$-algebras. Dokl. Akad. Nauk, Ross. Akad. Nauk. 2, 3-7 (2003)

8. Ayupov, Sh.A., Rakhimov, A.A., Usmanov, Sh.M.: Jordan, Real and Lie Structures in Operator Algebras (Monograph). Kluwer, Dordrecht, The Netherlands (1997)

9. Ayupov, Sh.A., Azamov, N.A.: Commutators and Lie isomorphisms of skew elements in prime operator algebras. Comm. Algebra 24(4), 1501-1520 (1996)

10. Inoue, A.: Tomita-Takesaki Theory in Algebras of Unbounded Operators, Lecture Note Math. N1699. Springer, Berlin Heidelberg New York (1998)

11. Li, B.R.: Real Operator Algebras (Monograph). World Scientific, Singapore (2002)

12. Sarymsakov, T.A., Goldshtein, M.Sh.: On partially ordered involutory algebras. Dokl. Akad. Nauk SSSR 228(2), 306-309 (1979)

13. Sarymsakov, T.A., Ayupov, Sh.A., Hadjiev, Dj., Chilin, V.I.: Ordered Algebras (Monograph). Fan, Tashkent, Uzbekistan (1983)

14. Schmüdgen, K.: Unbounded Operator Algebras and Representation Theory. Birkhäuser-Verlag, Basel, Switzerland (1990)

15. Segal, I.: A non commutative extension of abstract integration. Ann. of Math. 57, 401-457 (1953)

16. Størmer, E.: Jordan algebras of type I. Acta Math. 115(3,4), 165-184 (1966)

17. Yeadon, F.J.: Convergence of measurable operators. Proc. Camb. Phil. Soc. 74, 257-268 (1973) 